\title{
ESTUDIO QUÍMICO DE LOS TALLOS DE CINCHONA PUBESCENS
}

\section{CHEMICAL STUDY OF CINCHONA PUBESCENS STEMS}

\author{
Karin Loayza O. ${ }^{1}$, Brás H. de Oliveira ${ }^{2}$, Elena Cóndor C. ${ }^{1}$ y \\ Víctor Reyna P. ${ }^{1}$ \\ ${ }^{1}$ Facultad de Ciencias, Universidad Nacional de Ingeniería, \\ Av. Túpac Amaru 210, Lima 25, Perú; \\ 2 Departamento de Química, Universidad Federal de Paraná, \\ Curitiba -PR, Brasil- CEP 81.531-990, Brasil.
}

\section{RESUMEN}

De los tallos de Cinchona Pubescens que fueron colectados en la localidad de Yuracmallo (a 1 380 msnm, distrito de San Juan de Oro, Provincia de Sandia, Dpto. Puno) se aisló la quinina, que fue identificada mediante sus espectros de masas, $R M N{ }^{1} \mathrm{H}, \mathrm{RMN}^{13} \mathrm{C}$ y ${ }^{1} \mathrm{H}-{ }^{1} \mathrm{H}$ COSY.

Además se realizó el análisis cualitativo de sus metabolitos secundarios.

Palabras clave: Quinina; Cinchona pubescens; alcaloides quinolínicos.

\section{ABSTRACT}

From the stems of the Cinchona pubescens, wich were collected in the locality Yuracmallo (Province of Sandia, Puno), quinine was obtained, wich was identified by the mass spectra, $\mathrm{RMN}^{1} \mathrm{H}$, $\mathrm{RMN}^{13} \mathrm{C}$ y ${ }^{1} \mathrm{H}-{ }^{1} \mathrm{H}$ COSY. Furthermore, the qualitative analysis of the secondary metabolites was done.

Keywords: Quinine; Cinchona pubescens; quinoline alkaloids.

\section{INTRODUCCION}

El "árbol de la quina" o "cascarilla" símbolo en el escudo nacional de nuestra riqueza vegetal, comprende especies del genero Cinchona, entre ellas Cinchona calisaya, Cinchona officinalis y Cinchona pubescens.

Las dos especies más estudiadas son Cinchona calisaya, Cinchona officinalis, sus alcaloides han sido aislados y caracterizados por HPLC [1]. Se han aislado más de 25 alcaloides siendo la quinina y quinidina los alcaloides más importantes. Entre estos dos se ha obtenido la mayor producción comercial: al año 1988 la producción estimada era de 300 a 500 toneladas por año, lo cual significa que debieron procesar entre 5 a 10 mil toneladas de corteza de Cinchonas [2].

La importancia de las Cinchonas desde el siglo XVII se debió al uso de la corteza y, posteriormente, de sus alcaloides, en particular de la quinina, en el tratamiento de la malaria. Más adelante, hace ya varias décadas, la síntesis de compuestos alternativos a la quinina desplazó el interés por las cortezas de "quinas". Sin embargo en los últimos años ha surgido un renovado interés por estas plantas debido a que es el único compuesto antimalárico del que no se ha reportado resistencia del parásito Plasmodium falciparum y, por ello, se utiliza para combatir la malaria resistente al medicamento de síntesis cloroquina [2].

Los extractos de esta planta fueron introducidos como agentes médicos en el siglo XVII en Europa, luego que la condesa de Chinchón, esposa del virrey español en el Perú, fuera tratada y curada de la malaria. Linneo introdujo la denominación latina Cinchona en honor de dicha condesa en el año 1638 (Epperson et, al., 1995).

El uso de plantas del género Cinchona fue muy difundido y explotado en particular por sus propiedades febrífugas y antipalúdicas, por la presencia de alcaloides en la corteza, siendo explotadas en las expediciones llamadas cinchoneras. 
En el Perú se bebe la decocción en casos de arritmias cardiacas, fiebre, calambres musculares, resfríos e indigestión. La etnia campa-asháninka, pertenecientealaAmazonia peruana, emplea la decocción de corteza en el tratamiento de paludismo, reumatismo y diarreas. En la Amazonia Boliviana se emplea la corteza raspada y hervida con alcohol, como remedio antidiarreico.

Las últimas publicaciones referidas a Cinchona pubescens tratan del aislamiento e identificación de los alcaloides en diversos órganos de la planta obtenidos mediante cultivo celular [3].

Los alcaloides quinina $\left(1^{a}\right)$ y quinidina (1b), en la corteza de Cinchona se encuentran metoxilados: cinconidina (1c) y cinconina (1d), figura 1. En la estructura de estos alcaloides quinolínicos se ha considerado la numeración de Rabe, la cual es la más utilizada en la literatura química y farmacológica [2], y es la que utilizaremos en este trabajo.

El análisis cualitativo realizado por Hoet, Gómez y Kanamori (1980) [4] mediante el método de Bruselas - 1948 proporciono $0,02 \%$ y $0,08 \%$ de los alcaloides totales en dos muestras de Cinchona pubescens, colectada en la Convención (Cusco) y en San Ignacio (Cajamarca).
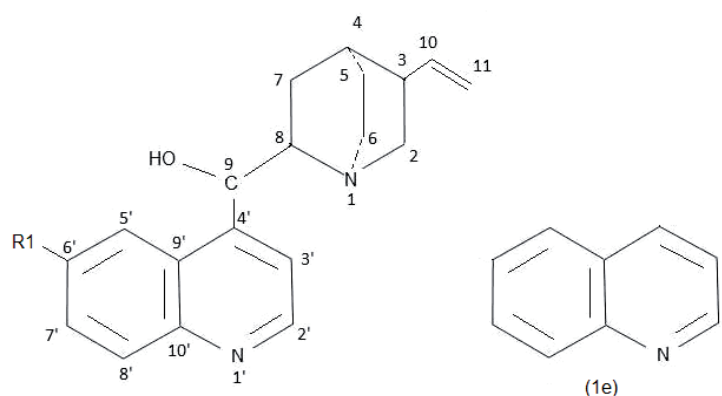

(1e)
R1 C-8
C-9
C-3

1a quinina $\quad \mathrm{OMe} \beta H(\mathrm{~S}) \quad \alpha H(R) \quad \alpha H(R)$

1b quinidina $\quad \mathrm{OMe} \quad \alpha \mathrm{H}(\mathrm{R}) \quad \beta H(\mathrm{~S}) \quad \alpha H(R)$

1c cinconidina $\quad H \quad \beta H(S) \quad \alpha H(R) \quad \alpha H(R)$

1d cinconina $\quad H \quad \alpha H(R) \quad \beta H(S) \quad \alpha H(R)$

1e estructura básica de los alcaloides quinolínicos.

Figura 1. Principales alcaloides presentes en corteza de Cinchona.

\section{PARTE EXPERIMENTAL}

El espectro de masas se registró en un espectrómetro de masas acoplado a cromatografía liquida, Applied Biosystems, modelo API300 (Laboratorio Central de Salud Publica de Paraná- LACEN/PR - Brasil); los espectros de Resonancia Magnética Nuclear de Hidrógeno y Carbono, ${ }^{1} \mathrm{H}-{ }^{1} \mathrm{H}$ COSY, se obtuvieron en un espectrómetro Brucker DRX400 (400MHz) (Laboratorio de Productos Naturales de la Universidad Federal de Minas Gerais - Brasil)

Materia Vegetal

La muestra de Cinchona pubescens fue colectada en Yuracmallo (1 380msnm) ubicada en el distrito de San Juan de Oro (Provincia de Sandia, Dpto. Puno) el 12 de Octubre de 2005, por la Blga. Joaquina Albán C. y nos fueron entregadas en estado seco (estufa a $35^{\circ} \mathrm{C}, 20$ horas -2 días) en mayo de 2006. Esta muestra está depositada en el Herbario San Marcos (JAC 15821) del Museo de Historia Natural de la Universidad Nacional de San Marcos. Lima.

Los tallos se cortaron con tijera de podar y se pulverizó a grano fino en una picadora-molino "Moulinex" (750 W), hasta obtener malla $500 \mu \mathrm{m}$.

\section{Análisis cualitativo (marcha fitoquímica)}

Los tallos de Cinchona pubescens se analizaron mediante los procedimientos de Rondina \& Coussio (1969) [5] y de Miranda (2002) [6] encontramos que contienen: Alcaloides $(+++)$, aminogrupos primarios y/o secundarios $(+)$, grupos fenólicos libres $(+)$, catequinas $(+)$, taninos $(++)$, triterpenos y esteroides $(+)$, quinonas, antraquinonas 0 antranoles (+), flavonoides y saponinas. Estos resultados se confirmaron con las pruebas específicas de Villacrez (1995) [2] .

\section{Aislamiento de la Quinina Extracción y separación *}

El material seco y pulverizado (150g) se "desengrasó" con n-hexano $(12 \times 300 \mathrm{ml})$ y después de dejar secar al ambiente se introdujo en un cartucho de tocuyo que se dispuso en el cuerpo central del equipo soxhlet. Luego la muestra se humedeció con una solución acuosa de amoniaco $15 \mathrm{~N}(200 \mathrm{ml})$ y se dejó en reposo durante dos horas. Sobre la muestra

*Esta parte del trabajo se realizó en el laboratorio de Productos Naturales de la Facultad de Ciencias-Universidad Nacional de Ingeniería 
alcalinizada, dentro del cuerpo central del soxhlet, se adicionó $200 \mathrm{ml}$ de una mezcla de metanol-cloroformo (1:1) y se dejó macerar durante 24 horas. La solución adquirió un color marrón oscuro.

En el balón del equipo soxhlet se dispuso $900 \mathrm{ml}$ de metanol-cloroformo-acetato de etilo (1:1:4), y se coloco a reflujo 6 h/día, durante 35 días, hasta que el solvente en el cuerpo central del soxhlet dio test negativo de Mayer.

El extracto orgánico se concentró en el rotavapor $\left(40^{\circ} \mathrm{C}, 300-400 \mathrm{mbar}\right)$ hasta casi obtener sequedad. El extracto orgánico fue tratado con acido sulfúrico $0,5 \mathrm{~N} \quad(4 \times 200 \mathrm{ml})$, para formar las sales de alcaloides; y la solución ácida fue lavada con cloroformo (2x350ml).

A continuación la solución ácida, fue alcalinizada con una solución de amoniaco $7,5 \mathrm{~N}$ $(36 \mathrm{ml})(\mathrm{pH}$ final $=9)$, realizando la separación de los alcaloides mediante su extracción con cloroformo $(35 \times 100 \mathrm{ml})$. El extracto orgánico se concentró en el rotavapor $\left(30^{\circ} \mathrm{C}, 300-400 \mathrm{mbar}\right)$ hasta lograr casi sequedad. Sobre el residuo de alcaloides se agregó $100 \mathrm{ml}$ de cloroformo y se volvió a concentrar a sequedad en el rotavapor, $\left(30^{\circ} \mathrm{C}, 300-400 \mathrm{mbar}\right)$, repitiéndose esta operación un total de cuatro veces. Esto tiene por objeto eliminar las bases volátiles (amoniaco, $\mathrm{NH}_{3(\mathrm{~g})}$ ) que estuvieran disueltas en el extracto.

La eliminación de los solventes orgánicos en el rotavapor, $\left(30^{\circ} \mathrm{C}, 300-400 \mathrm{mbar}\right)$ hasta obtener sequedad nos proporcionó el extracto bruto de alcaloides, en forma de un sólido de color marrón oscuro $(1,6 \mathrm{~g})$. El análisis por $\mathrm{CCF}^{*}$ nos indicó que el extracto contenía quinina.

\section{Purificación cromatográfica}

La purificación del extracto bruto de alcaloides -EBA $(1,6 \mathrm{~g})$ se realizó mediante dos técnicas cromatográficas: Primero por cromatografía en columna a vacío-CCV del EBA, seguida de cromatografía en capa delgada centrifugada - CCDC (4 ensayos consecutivos) de las fracciones purificadas (que contenían el alcaloide quinina).

\section{Cromatografía en columna a vacío, CCV}

La cromatografía en columna a vacío del EBA $(1,6 \mathrm{~g})$ se realizó en una columna $(\mathrm{h}=38 \mathrm{~cm}$, $\phi=5 \mathrm{~cm})$ que contenía $90 \mathrm{~g}(\mathrm{~h}=15 \mathrm{~cm}) \mathrm{de}$

*CCF: placas de sílica gel 60 F 254, el eluente: cloroformoacetona-amoniaco (10:17:1) Revelador: UV; Rf (quinina= 0,62). absorbente sílica gel 60 para CC (porosidad 0,040-0,063).

El EBA se disolvió en $5 \mathrm{ml}$ de cloruro de metileno y se dispuso en la columna, agregándose como eluente acetato de etilo (200ml, fracción $\mathrm{N}^{\circ} 1$ ), seguido de $200 \mathrm{ml}$ de mezclas de acetato de etilo con cantidades crecientes de metanol (10\%,fracción $\mathrm{N}^{\circ} 2$; $20 \%$,fracción $N^{\circ} 3$; etc ), hasta agregar metanol puro ( fracción $\mathrm{N}^{\circ} 12$ ), recibiéndose las fracciones en balones de $500 \mathrm{ml}$. Todas las fracciones tenían una coloración amarilla y se llevaron a sequedad en el rotavapor $\left(40-50^{\circ} \mathrm{C}\right)$. De las 12 fracciones obtenidas, la quinina se presentó parcialmente pura en las fracciones $\mathrm{N}^{\circ} 3$ y $\mathrm{N}^{\circ} 4$ con un total de $741 \mathrm{mg}$ (eluentes: $20 \%$ y $30 \%$ de metanol en acetato de etilo, respectivamente).

\section{Cromatografía en capa delgada centrifugada, CCDC}

Las fracciones purificadas por CCV (741 mg) se sometieron a cromatografía en capa delgada centrifugada CCDC- $\mathrm{N}^{\circ} 1$, obteniéndose quinina (fracción $\mathrm{N}^{\circ} 16-19,231 \mathrm{mg}$ ), la cual se sometió a una segunda cromatografía CCDC- $\mathrm{N}^{\circ} 2$, obteniéndose quinina (fracción $\mathrm{N}^{\circ}$ 3-7, $180 \mathrm{mg}$ ), la cual se sometió nuevamente a una tercera cromatografía CCDC- $\mathrm{N}^{\circ} 3$, obteniéndose quinina (fracción $\mathrm{N}^{\circ} 3-5,133 \mathrm{mg}$ ), la cual, finalmente, fue purificada mediante CCDC- $\mathrm{N}^{\circ} 4$, obteniéndose quinina pura $(14 \mathrm{mg})$ en las fracciones $\mathrm{N}^{\circ}$ 17-26.

Estas cuatro secuencias cromatográficas se resumen a continuación:

\section{CCDC No 1}

i.- Muestra a purificar: fracciones $\mathrm{N}^{\circ} 3-4$ (741 mg) obtenido de CCV.

ii.- Resultados: se obtuvieron 23 fracciones de $15 \mathrm{ml} \mathrm{c} / \mathrm{u}$. las fracciones $\mathrm{N}^{\circ} 16-19$ (231 mg) contenían quinina impura*.

\section{CCDC No 2}

i.- Muestra a purificar: fracciones $\mathrm{N}^{\circ} 16-19$ (231 mg) obtenido de CCDCN ${ }^{\circ} 1$.

ii.- Resultados: se obtuvieron 20 fracciones de $10 \mathrm{~mL}$ c/u. Las fracciones $\mathrm{N}^{\circ} 3-7$ (180mg) contenían quinina impura*.

\section{CCDC No 3}

i.- Muestra a purificar: fracciones $\mathrm{N}^{\circ}$ 3-7 (180mg) obtenido de $\mathrm{CCDCN}^{\circ} 2$.

*EI análisis de las fracciones se realizó mediante CCF: adsorbente: Placas de aluminio, Kieselgel 60F-254, 0,2mm; eluente acetona: $\mathrm{CHCl} 3: \mathrm{NH} 315 \mathrm{~N}(17: 10: 1)$. 
ii.- Resultados: se obtuvieron 32 fracciones de $10 \mathrm{ml} \mathrm{c} / \mathrm{u}$. Las fracciones $\mathrm{N}^{\circ} 3-5$ (133mg) contenían quinina impura.

\section{CCDC No 4}

i.-- Muestra a purificar: fracciones $\mathrm{N}^{\circ}$ 3-5 (133mg) obtenido de CCDCN ${ }^{\circ} 3$.

ii.- Resultados: se obtuvieron 20 fracciones de $10 \mathrm{~mL} \mathrm{c} / \mathrm{u}$. Las fracciones $\mathrm{N}^{\circ} 17-26(14 \mathrm{mg})$ contenían quinina pura**.

Las condiciones de trabajo y el procedimiento de separación fueron similares para las cuatro eluciones cromatográficas. A continuación se describe lo realizado para la purificación final de la quinina (CCDC-N4).

\section{Condiciones de trabajo}

l.- Muestra: fracciones $\mathrm{N}^{\circ} 3-5$ (133mg) obtenida en CCDC N ${ }^{\circ} 3$.

ii.- Placa de vidrio $=24 \mathrm{~cm}$, sílica gel grado 7749 con yeso e indicador de fluorescencia; espesor de la placa $1 \mathrm{~mm}$. (Observación: sólo en la primera placa, $\operatorname{CCDC} \mathrm{N}^{\circ} 1$, el espesor fue de $2 \mathrm{~mm}$ ).

iii.-Eluente ${ }^{* * *}$ :acetatodeetilo: $\mathrm{CH}_{2} \mathrm{Cl}_{2}: \mathrm{NH}_{3(\mathrm{ac})} 15 \mathrm{~N}$ $(10: 1: 0,2)$ y metanol.

iv.- Revelador: Luz UV (254y 360nm); se dispuso como patrón quinina pura.

\section{Procedimiento}

İ.- Se humedeció la placa haciéndose pasar el eluente, luego se adicionó la muestra disuelta en $0,5 \mathrm{ml}$ de etanol.

ii.- Se adicionó $320 \mathrm{ml}$ de acetato de etilo:diclorometano: $\mathrm{NH}_{3(\mathrm{ac})} 15 \mathrm{~N}(10: 1: 0,2)$.

iii.- Finalmente, se adicionó $50 \mathrm{ml}$ de metanol, con la finalidad de lavar la placa.

iv.- Se colectaron 20 fracciones de $10 \mathrm{ml}$ cada una (tubos $100 \times 15 \mathrm{~mm}$, la mayoría de las cuales eran incoloras. Las fracciones $\mathrm{N}^{\circ} 17-26$ (14mg) proporcionaron un sólido blanco, cuyo análisis por $\mathrm{RMN}^{1} \mathrm{H}$ y CCF nos indica que se trata de la quinina pura.

\footnotetext{
** De acuerdo a CCF y RMN1H.

*** Los eluentes para las otras elusiones cromatográficas fueron: CCDCN ${ }^{\circ}$ : $\mathrm{CH} 2 \mathrm{Cl} 2: \mathrm{NH} 3(\mathrm{ac}) 15 \mathrm{~N}(10: 0,3) ; \mathrm{CH} 2 \mathrm{Cl} 2: \mathrm{AcEt}: \mathrm{NH} 3(\mathrm{ac})$ $15 \mathrm{~N}(75: 75: 4,5)$.

$\mathrm{CCDCN}^{\circ}$ 2: Metanol:AcEt: NH3(ac)15N(2:8:0,1) y metanol. $\mathrm{CCDCN}^{\circ} 3$ : AcEt: $\mathrm{NH} 3(\mathrm{ac}) 15 \mathrm{~N}(5: 0,2)$ y methanol.
}

\section{RESULTADOS Y DISCUSION}

(Identificación espectroscópica de la
quinina)

\section{Espectro de Masas. Espectro 1}

Características del espectro: $\mathrm{m} / \mathrm{z} 325.5$ (ión molecular, $\left.\mathrm{M}^{+}+1\right), 304,4\left(\mathrm{M}^{+}-\mathrm{H}_{2} \mathrm{O}\right): 255,1$; 188,$1 ; 172,1$ (pico base), 160,1; 81,1.

Los fragmentos que corresponden a los iones $\mathrm{m} / \mathrm{z} 307,4 ; \mathrm{m} / \mathrm{z} 188,1$ y $\mathrm{m} / \mathrm{z} 172,1$ son los siguientes:
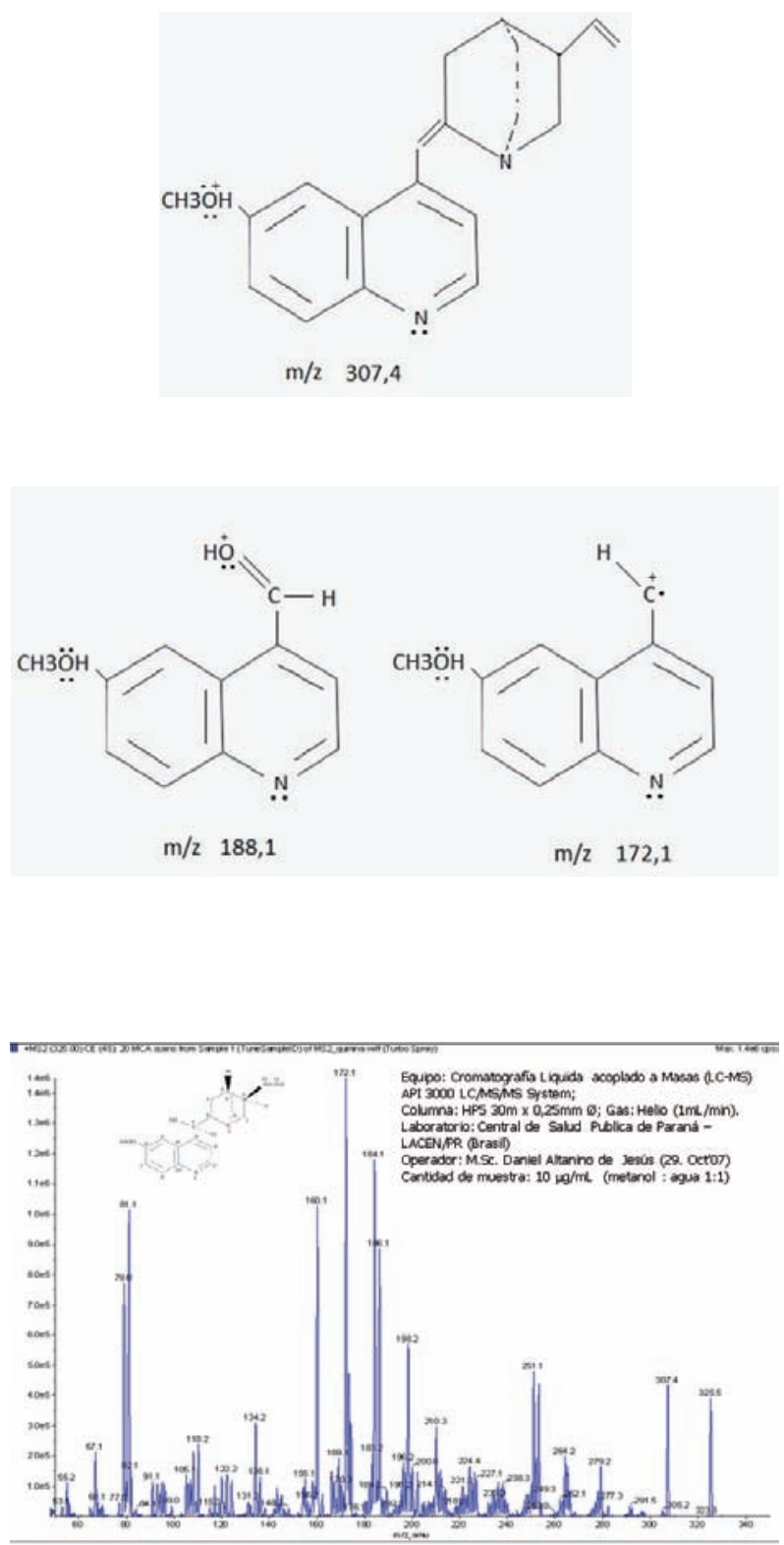

Espectro 1. Espectro de masas de la quinina aislada de los tallos de Cinchona pubescens. 


\section{Espectro de RMN'1 H (400 MHz en $\mathrm{CDCl}_{3}$ ). Espectro 2}

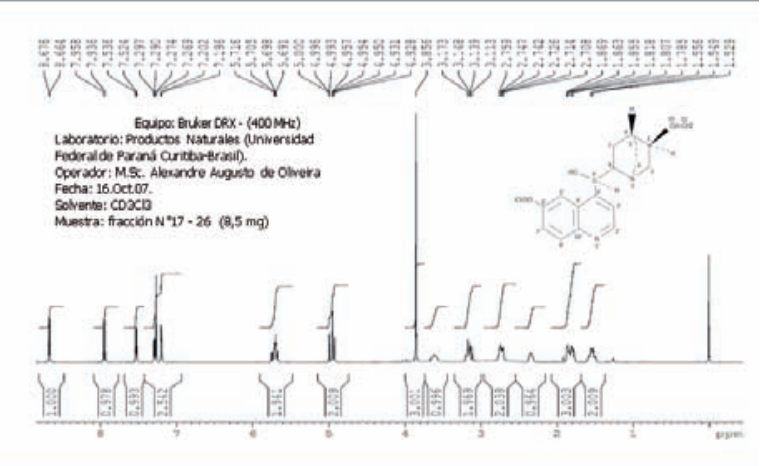

Espectro 2. Espectro de RMN ${ }^{1} \mathrm{H}(400 \mathrm{MHz})$ de la quinina aislada de los tallos de Cinchona pubescens

\section{Características del espectro:}

$\delta(\mathrm{ppm}): 1,52 \mathrm{ppm}(\mathrm{m}, \mathrm{H}-7 \mathrm{eq}) ; 1,54 \mathrm{ppm}(\mathrm{m}$, $\mathrm{H}-6 \mathrm{eq}) ; 1,71 \mathrm{ppm}(\mathrm{m}, \mathrm{H}-6 \mathrm{ax}) ; 1,84 \mathrm{ppm}$ (m, H- 4); 1,86 ppm (m, H-7ax); 2,70 ppm (s ancho, $\mathrm{H}-3) ; 2,73 \mathrm{ppm}(\mathrm{m}, \mathrm{H}-2 \mathrm{eq}) ; 2,75 \mathrm{ppm}$ (m, H-5 eq); 3,12 ppm (m, H-2ax); 3,15 ppm (m, H-8); 3,17 ppm (t ancho, H-5ax); 3,85 ppm (s, $\left.-\mathrm{OCH}_{3}\right) ; 4,95$ ppm (d, H-11 gem); 4,97 ppm (d, H-11 gem); 5,69 ppm (d, H-9); 5,70 ppm (m, $\mathrm{H}-10$ ); 7,19 ppm (s, H-5'); 7,28 ppm (d, H-7'); 7,52 ppm (d, H-3'); 7,94 (d, H-8'); 8,66 ppm (d, H-2').

Este espectro corresponde a lo esperado para la estructura de la quinina y es similar a lo publicado precedentemente [2] [8].

Espectro de $\mathrm{RMN}^{13} \mathrm{C}\left(400 \mathrm{MHz}\right.$ en $\mathrm{CDCl}_{3}$ ). Espectro 3

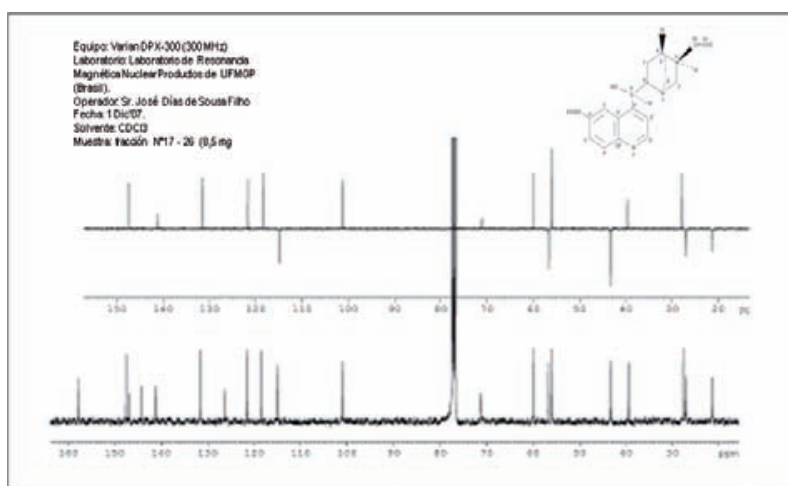

Espectro 3. Espectro de $\mathrm{RMN}^{13} \mathrm{C}$ de la quinina aislada de los tallos de Cinchona pubescens. a) Características del espectro DEPT-135

Señales hacia arriba (11):

i. Carbonos primarios $\left(\mathrm{CH}_{3}\right): \delta(\mathrm{ppm}): 55,8$ $\left(-\mathrm{OCH}_{3}\right)$

ii. Carbonos terciarios (CH): $\delta$ (ppm): 27,7 $\left(\mathrm{C}_{4}\right) ; 39,5\left(\mathrm{C}_{3}\right) ; 59,9\left(\mathrm{C}_{8}\right) ; 71,1\left(\mathrm{C}_{9}\right) ; 101,1$ $\left(\mathrm{C}_{5}\right)$; 118,4 $\left(\mathrm{C}_{3,}\right) ; 121,6\left(\mathrm{C}_{7}\right)$; 131,6 ( $\left.\mathrm{C}_{8}\right)$; $141,1\left(C_{10}\right) ; 147,5\left(C_{4}\right)$.

Señales hacia abajo (5): carbonos secundarios $\left(\mathrm{CH}_{2}\right): \delta(\mathrm{ppm}): 2,13\left(\mathrm{C}_{7}\right) ; 27,1$ $\left(\mathrm{C}_{6}\right) ; 43,3\left(\mathrm{C}_{5}\right) ; 56,6\left(\mathrm{C}_{2}\right) ; 114,8\left(\mathrm{C}_{11}\right)$.

b) Desacoplamiento de ${ }^{1} \mathrm{H}$ de banda ancha. Características del espectro

En este tipo de espectro aparecen todos los carbonos:

$\delta$ (ppm): 21,3 $\left(\mathrm{CH}_{2}\right) ; 27,1\left(\mathrm{CH}_{2}\right) ; 27,7(\mathrm{CH})$; $43,3\left(\mathrm{CH}_{2}\right) ; 55,8\left(\mathrm{O}-\mathrm{CH}_{3}\right) ; 56,6\left(\mathrm{CH}_{2}\right) ; 59,9$ (CH); 71,1 (CH); 101,1 (CH-aromático); 114,8 $\left(\mathrm{CH}_{2}\right.$ vinilo): 119,4 (CH-aromático); 121,6 (CH-aromático);126,4(C-aromático); 131,5 (CH-aromático); 141,1 (CH-vinilo); 144,2 (C-aromático); 146,9 (C-aromático); 147,5 (CH-aromático); 157,8 (C-aromático).

La asignación de los valores de desplazamiento químico son análogos a lo publicado precedentemente [2].

\section{Espectro 2D ${ }^{1} \mathrm{H}-{ }^{1} \mathrm{H}$ COSY $(400 \mathrm{MHz}$ en $\mathrm{CDCl}_{3}$ ). \\ Espectro 4}

\section{Características del espectro}

\begin{tabular}{|c|c|}
\hline $\mathbf{H}(\delta \mathbf{p p m})$ & Hidrógenos \\
\hline $\mathrm{H}-7$ eq $(1,52)$ & $\begin{array}{l}\mathrm{H}-6 \mathrm{ax}(1,71) ; \mathrm{H}-4(1,84) ; \\
\mathrm{H}-7 \mathrm{ax}(1,86)\end{array}$ \\
\hline H-6eq $(1,54)$ & $\begin{array}{l}\mathrm{H}-5 \mathrm{eq}(2,75) ; \mathrm{H}-8(3,15) ; \\
\mathrm{H}-5 \mathrm{ax}(3,17)\end{array}$ \\
\hline H-6ax $(1,71)$ & $\mathrm{H}-5 \mathrm{eq}(2,75), \mathrm{H}-5 \mathrm{ax}(3,17)$ \\
\hline $\mathrm{H}-4(1,84)$ & $\mathrm{H}-3(2,70)$ \\
\hline $\mathrm{H}-7$ ax $(1,86)$ & $\mathrm{H}-8(3,15)$ \\
\hline $\mathrm{H}-2$ eq $(2,73)$ & $\mathrm{H}-11$ gem $(4,95-4,97)$ \\
\hline $\mathrm{H}-8(3,15)$ & $\mathrm{H}-9(5,69)$ \\
\hline$-\mathrm{OCH} 3(3,85)$ & $H-5^{\prime}(7,19)$ \\
\hline $\mathrm{H}-11$ gem $(4,95-4,97)$ & $\mathrm{H}-10(5,70)$ \\
\hline $\mathrm{H}-9(5,69)$ & $\mathrm{H}-3^{\prime}(7,52)$ \\
\hline$-7^{\prime}(7,28)$ & H - 8' $(7,94)$ \\
\hline $\mathrm{H}^{\prime}(7,52)$ & H- 2' $(8,66)$ \\
\hline
\end{tabular}




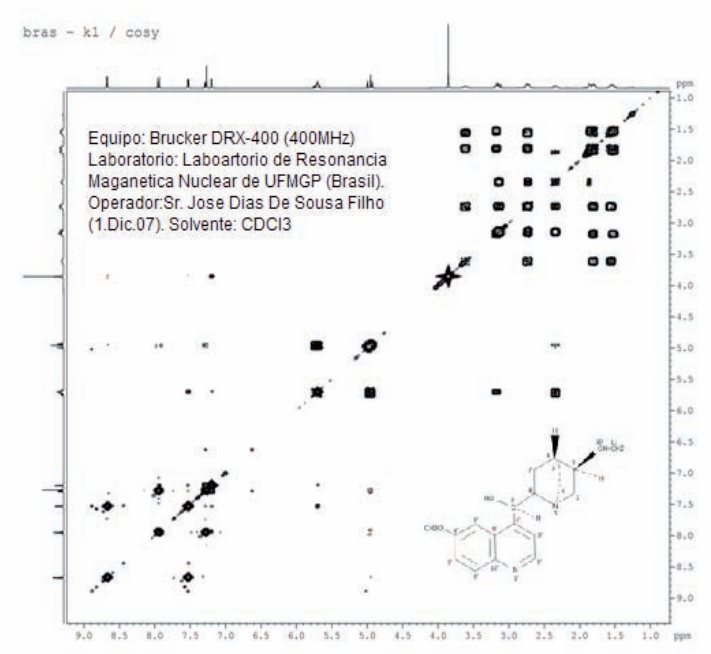

Espectro 4. Espectro 2D ${ }^{1} \mathrm{H}-{ }^{1} \mathrm{H}$ COSY $(400 \mathrm{MHz})$ de la quinina aislada de los tallos de Cinchona pubescens.

\section{CONCLUSIONES}

A partir de los tallos de Cinchona pubescens se aisló la quinina, la cual se identifico mediante sus espectros de masas, $R M N^{1} H, R^{\prime} N^{13} \mathrm{C}$ y ${ }^{1} \mathrm{H}-{ }^{1} \mathrm{H}$ COSY.

Se realizó el análisis cualitativo de los metabolitos secundarios (marcha fitoquímica) presentes en los tallos de la Cinchona pubescens según el procedimiento de Rondina Coussio (1969) y Miranda (2002), habiéndose verificado la presencia de: alcaloides, aminogrupos primarios y/o secundarios, grupos fenólicos libres, taninos, triterpenos y esteroides, quinonas, antraquinonas, antronas o antranoles, saponinas, flavonoides, leucoantocianidinas, catequinas, flavonoides, saponinas, glicósidos cardiotónicos, aminoácidos libres o aminas en general.

\section{AGRADECIMIENTOS}

Agradecemos a la bióloga Joaquina Albán (profesora principal y jefa del departamento de Etnobotánica y Botánica Económica del Museo de Historia Natural de la Universidad Nacional de San Marcos) por habernos proporcionado el material vegetal y por su determinación botánica; a la M.Sc Virginia Torpoco Carmen por la elucidación de los espectros $\mathrm{RMN}^{1} \mathrm{H}$, $\mathrm{RMN}^{13} \mathrm{C}$, al Instituto de Investigación de la UNI y al Instituto de Investigación de la Facultad de Ciencias (UNI) por el apoyo económico que nos brindaron para la realización de todo este trabajo de investigación.

\section{REFERENCIAS}

[1] Keene, A. et al., J. of Chromatography, 260,123-128 (1983). "Investigation of Cinchona Leaf Alkaloids by High Performance Liquid Chromatography". Content of Callus Cultures of Cinchona pubescens".

[2] Verpoorte, R. et al., "Cinchona Alkaloids", in: Brossi, A. Ed., The Alkaloids Chemistry and Pharmacology, vol. 34, Academia Press, 1988, pag. 358-369.

[3] Verpoorte, R. et al. J. of Medicinal, vol. 46, pag. 15-18 (1982). "The effects of Plant Growth Regulators and Culture Conditions on the Growth and the Alkaloid Content of Callus Cultures of Cinchona pubescens".

[4] Hoet, P. Gómez, a. Kanamori, C. et al., Estudio Cuantitativo de los alcaloides en Cinchona (Rubiaceae) del Perú. Boletín Soc. Quim. (Perú); 46, 298-309 (1980).

[5] Rondina R. y J. Coussio. Revista de Investigación Agropecuaria. INTA, Buenos Aires- Argentina, 1969, Serie 2. vol. VI. No 22. pag. 351-366.

[6] Miranda, M., Farmacognosia y Productos Naturales, Universidad de La Habana, Cuba, 2002, pág. 41-49.

[7] Villacrez, O., Víctor, Bioactividad de Plantas amazónicas, Ed., Ab Yala, Ecuador, 1995, pag. 154-171.

[8] Mills, T. et al., Intrumental Data for Drug Analysis, vol. 4, Taylor \& Francis. Group CRC Press, $3^{\circ}$ Ed., 2006, pag. 2744-2745.

E-mail: jessy_uni9@hotmail.com 